\title{
Cambios en el control metabólico de los pacientes diabéticos tipo 2 de un centro de salud
}

\author{
Pedro Domínguez Sánchez-Migallón.
}

Médico de Familia. Centro de Salud Manzanares II. Gerencia de Atención Integrada de Manzanares. Servicio de Salud de Castilla-La Mancha (España).

Correspondencia: Pedro Domínguez Sánchez-Migallón. C/ Dr. Fleming, 25. C.P. 13200. Manzanares - Ciudad Real (España). Correo electrónico: pdsanchez@sescam.jccm.es

Recibido el 18 de septiembre de 2014.

Aceptado para su publicación el 11 de diciembre de 2014

\section{RESUMEN}

Objetivo: Conocer los cambios producidos en un período de cuatro años en el control de los pacientes diabéticos y en el uso de la medicación en la práctica habitual.

Diseño del estudio: Estudio descriptivo, observacional, transversal.

Emplazamiento: Zona de Salud Manzanares II (Manzanares - Ciudad Real - España).

Participantes: Pacientes diabéticos de ambos sexos de la Zona de Salud.

Mediciones principales: Se recogieron datos sociodemográficos, factores de riesgo cardiovascular, repercusión macro y microvascular de la diabetes, valores analíticos (glucídicos, lipídicos) y tensionales, así como los fármacos empleados en el tratamiento de diabetes, dislipemia y con efecto antiagregante/anticoagulante. Los datos fueron comparados con los del estudio observacional realizado en 2010 .

Resultados: El control metabólico (HbA1c) ha mejorado en 2014: 7,09\% (DE: 1,17) respecto a 2010: 7,22\% (DE: 1,21), siendo significativo en el porcentaje de valores más elevados de HbA1c ( $\geq 8 \%$ y $\geq 10 \%$ ). El porcentaje de $\mathrm{HbA} 1 \mathrm{c} \leq 7 \%$ ha pasado del $50,5 \%$ al $53,7 \%$. Los mayores descensos se han producido en los parámetros lipídicos: colesterol total $(p<0,001)$, LDL-colesterol $(p<0,001)$ y microalbuminuria $(p<0,01)$. El porcentaje de $\mathrm{HbA} 1 \mathrm{c} \leq 7 \%$ y LDL-colesterol $\leq 100 \mathrm{mg} /$ dl ha pasado del $13,1 \%$ al $21,7 \%(p<0,01)$. Ha disminuido el uso de sulfonilureas $(p<0,01)$ y aumentado el de IDDP-4 $(p<0,001)$, estatinas $(p<0,05)$ y de ácido acetilsalicílico $(p<0,01)$.

Conclusiones: En el periodo de cuatro años ha mejorado el control metabólico glucídico, siendo significativo el cambio en el patrón lipídico. Ha disminuido el uso de sulfonilureas y ha aumentado el uso de IDPP-4, estatinas y ácido acetilsalicílico.

PALABRAS CLAVE: Diabetes Mellitus. Control. Antidiabéticos.

\section{ABSTRACT}

\section{Changes in the metabolic control of type 2 diabetic patients in a health center}

Objective: To know the changes achieved in a period of four years in the control of diabetic patients, and the changes in the use of medication in routine practice.

Study design: Cross-sectional descriptive study.

Setting: Primary Care Centre of Manzanares II (Manzanares - Ciudad Real - Spain).

Participants: Diabetic patients of both sexes in the Health Zone.

Measurements: Sociodemographic data, cardiovascular risk factors, macro and microvascular impact of diabetes, blood pressure and analytical parameters (glucidic, lipidic), as well as drugs used in the treatment of diabetes and dyslipidemia, and antiplatelet/anticoagulant drugs were collected. These data were compared with those of the cross-sectional descriptive study conducted in 2010.

Results: The metabolic control (HbA1C) has improved in 2014: $7.09 \%$ (SD:1.17) with regard to 2010: $7.22 \%$ (SD: 1.21), being significant in the percentage of higher values of $\mathrm{HbA1C}(\geq 8 \%$ and $\geq 10 \%$ ). The percentage of $\mathrm{HbA} 1 \mathrm{C} \leq 7 \%$ has increased from $50.5 \%$ to $53.7 \%$. The largest decreases have occurred in the lipid parameters: total cholesterol $(p<0.001)$, LDL-cholesterol $(p<0.001))$ and microalbuminuria $(p<0.01)$. The percentage of HbA1C $\leq 7 \%$ and LDL-cholesterol $\leq 100 \mathrm{mg} / \mathrm{dl}$ has increased from $13.1 \%$ to $21.7 \%(p<0,01)$. The use of sulfonylureas has decreased $(p<0.01)$ and those of IDDP-4 $(p<0.001)$, statins $(p<0.05)$ and acetyilsalicylic acid $(p<0.01)$ have increased.

Conclusions: The carbohydrate metabolic control has improved during a period of four years, with significant changes in lipid parameters. The use of sulfonylureas has decreased, and the use of IDPP-4, statins and acetylsalicylic acid has increased.

KEY WORDS: Diabetes Mellitus. Control. Antidiabetics. 


\section{INTRODUCCIÓN}

La prevalencia de diabetes conocida es variable, con un rango de valores desde del $6,36 \%$ de la población general en el Área de Atención Primaria Mancha-Centro ${ }^{1}$, al 10,7\% en $\geq 30$ años de CastiIla-La Mancha ${ }^{2}$, o al 7,8\% - con un 6\% de diabetes desconocida - en España del estudio Di@bet.es ${ }^{3}$.

La prevalencia de la diabetes mellitus tipo 2 (DM2) tiene tendencia a incrementarse, debido a los cambios alimenticios así como al envejecimiento poblacional, cambios en los criterios diagnósticos y menor mortalidad de los pacientes diabéticos ${ }^{4}$. En el paciente diabético tipo 2 se presentan de modo simultáneo diversos factores de riesgo cardiovascular (FRCV) - dislipemia, hipertensión y obesidadque incrementan el riesgo cardiovascular, siendo la asociación con la obesidad la que conlleva un mayor gasto sanitario ${ }^{5}$, y dificultando el control metabólico óptimo de estos pacientes hasta el punto de que, en los diabéticos tipo 2 incluidos en ensayos clínicos, solo se consigue el control en un máximo de dos tercios de los pacientes, según estimó Winocour ${ }^{6}$. Los principales motivos implicados en la no consecución de los objetivos de control en el paciente con DM2 son la inercia terapéutica, el incumplimiento terapéutico, los problemas inherentes al sistema sanitario y la historia natural de la enfermedad ${ }^{7}$.

Estudios observacionales realizados en España y en otros países muestran que existe un vacío entre las recomendaciones y la práctica clínica diaria ${ }^{8}$, por lo que hay que considerar que el grado de control de los pacientes diabéticos es bajo e inferior a lo deseable 9 , con cifras medias de $\mathrm{HbA} 1 \mathrm{c} \leq 7 \%$ entre el 41,3 y el $56,9 \%$, LDL-colesterol $(\leq 100 \mathrm{mg} / \mathrm{dl})$ entre el $5,9 \%$ y $30,6 \%$, y cifras tensionales $\leq 130$ / $\leq 80 \mathrm{~mm} \mathrm{Hg}$ ) entre el $7,8 \%$ y $38,3 \%^{10-15}$. Varios estudios indican que solo el $7-9 \%$ de los pacientes tienen todos los FRCV controlados ${ }^{8}$, siendo este porcentaje del $7,07 \%$ en los pacientes de nuestra Zona de Salud ${ }^{9}$.

Es conocido que en el tratamiento del paciente diabético puede ser necesario emplear varios fármacos (además de los que tienen efecto hipoglucemiante) ${ }^{16}$, que no son prescritos en nuestro medio en todos los casos en que estarían indicados: metformina en pacientes obesos sin contraindicación para su uso ${ }^{17} \mathrm{y}$ antiagregantes y estatinas ${ }^{17,18}$.

Un adecuado manejo de los fármacos y un abordaje global del riesgo cardiovascular ha demostrado (estudio STENO-2) ${ }^{19}$ una reducción de los eventos cardiovasculares [RR: $0,47(0,24-0,73) ; p=0,007]$ y de las complicaciones microvasculares. Hay que tener presente la comorbilidad existente en el paciente diabético cuando se realiza la indicación del tratamiento antidiabético ${ }^{20}$, lo que condiciona el empleo de un fármaco $u$ otro.

En algunos estudios se ha visto que tras 4 años de seguimiento por atención especializada a una cohorte de diabéticos, el porcentaje de pacientes con hemoglobina glicada $<7 \%$ se mantuvo estable, mejorando el control lipídico y tensional, pero para conseguir estos resultados las pautas de tratamiento se complicaron de manera significativa $^{21}$; así como en otro estudio ${ }^{22}$, igualmente en atención especializada, el porcentaje de pacientes con $\mathrm{HbA} 1 \mathrm{c}$ media $<7 \%$ se mantuvo estable $(30 \%)$, aumentando el porcentaje (al 25\%) con LDL-colesterol $<100 \mathrm{mg} / \mathrm{dl}$, y solo el $9,2 \%$ de los pacientes alcanzaron el objetivo combinado de $\mathrm{HbA} 1 \mathrm{c}<7 \%$ y LDL $<100 \mathrm{mg} / \mathrm{dl}$.

El objetivo principal del estudio es conocer el grado de control de los pacientes diabéticos tipo 2 de nuestra zona, siendo objetivos secundarios valorar si ha mejorado significativamente respecto al estudio previo (Estudio AZUER) ${ }^{9}$ realizado en 2010 y publicado en 2011, y valorar si han existido cambios en el empleo de fármacos antidiabéticos.

\section{SUJETOS Y MÉTODOS}

Se trata de un estudio observacional, descriptivo, transversal en el ámbito de Atención Primaria: Zona de Salud Manzanares II (que comprende la población de Membrilla y más del $50 \%$ de la población de Manzanares), con una población de 14.596 habitantes de $\geq 14$ años, siendo el periodo de estudio del 1 de mayo al 30 de junio de 2014.

La población analizada han sido los diabéticos tipo 2 de ambos sexos de la Zona de Salud (1.445 pacientes) identificados mediante historia clínica informatizada. Se han excluido los pacientes diabéticos tipo 1, desplazados o adscritos temporalmente al cupo médico y fallecidos - aunque figurasen en la base de datos -, así como aquellos en que los datos clínico-analíticos no se considerasen válidos. Para el cálculo del tamaño muestral se empleó la fórmula de proporciones en poblaciones finitas (considerando una prevalencia de diabetes del $25 \%$ ), obteniendo un tamaño muestral mínimo de 288 pacientes.

La selección de éstos se realizó por muestreo sistemático sobre el listado de diabéticos de cada uno de los cupos médicos. De cada paciente seleccionado se rellenó una hoja de recogida de datos (HRD), donde se registraron datos sociodemográfi- 
cos (sexo y edad), factores de riesgo cardiovasculares asociados, repercusión macro y microvascular de la diabetes tipo 2, valores analíticos y antropométricos (considerados como válidos si tenían una antigüedad $\leq 6$ meses). En concreto: glucemia basal, HbA1c, colesterol total, HDL y LDL colesterol, triglicéridos, tabaquismo, creatinina y microalbuminuria en orina de una micción ( $\geq 20 \mathrm{mg} / \mathrm{l})$. Se registraron las cifras de presión arterial (PA) de la última visita. También se recogieron los fármacos que tomaban los pacientes para el tratamiento de la DM, hipertensión arterial (HTA), dislipemia, antiagregantes o anticoagulantes. Los parámetros clínicos se determinaron para todos los pacientes en el laboratorio del hospital de referencia (Virgen de Altagracia de Manzanares).

Se han obtenido 337 HRD válidas. Para el estudio descriptivo de los datos se ha utilizado la media (DE) para las variables cuantitativas y la proporción para las variables cualitativas, y para el estudio analítico la comparación de medias mediante la $t$ de Student y la Chi-cuadrado para la comparación de porcentajes. Se han calculado los intervalos de confianza al 95\% (IC95\%).

Los valores obtenidos en este estudio se han comparado con los del Estudio $\mathrm{AZUER}^{9}$, realizado con una metodología similar sobre 297 historias clínicas de diabéticos de ambos sexos, para valorar los cambios que se han producido en el período 2010-2014.

El análisis estadístico se efectuó con el programa estadístico G-Stat 2.0.

\section{RESULTADOS}

La prevalencia conocida de DM2 en mayores de 14 años ha pasado del 8,5\% (IC95\%: 8,0-8,9) en 2010 al 9,9\% (IC95\%: 9,4-10,4) en 2014 ( $p<0,001)$. No hay diferencias significativas (aunque los valores son inferiores en 2014) en cuanto a la media de la edad y coexistencia de otros factores de riesgo cardiovascular (HTA, dislipemia, obesidad y tabaquismo). En cuanto a la afectación orgánica recogida en las historias, hay una disminución en la prevalencia, aunque sólo es significativa en nefropatía diabética ( $p<0,05)$, enfermedad cerebrovascular $(p<0,01)$, y neuropatía diabética $(p<0,001)$ (tabla 1).

El control metabólico ha mejorado en 2014, ya que si bien ha aumentado el valor medio de glucemia [141,0 mg/dl (DE: 31,4)], hay una tendencia al descenso, no significativo, en los valores medios de hemoglobina glicada desde $7,22 \%$ (DE: 1,21$)$ hasta
$7,09 \%$ (DE: 1,17), aunque sí hay significación en los valores más elevados $(\mathrm{HbA} 1 \mathrm{c} \geq 8 \%$ y sobre todo en $\geq 10 \%$ ) con $p<0,05$ y $p<0,01$, respectivamente (tabla 1).

Existe un descenso en los valores de colesterol total $(p<0,001)$ y LDL colesterol $(p<0,001)$ con aumento de niveles de HDL-colesterol $(p<0,01)$, así como en los valores medios de microalbuminuria (MAU) en orina de una micción $(p<0,01)$. Hay un descenso no significativo $(p=0,06)$ en la prevalencia de enfermedad renal crónica (tabla 1).

El porcentaje de pacientes con un adecuado control ha mejorado en todos los parámetros (excepto en presión arterial sistólica $<140 \mathrm{~mm} \mathrm{Hg}$ ), siendo estadísticamente significativo en colesterol total $\leq$ $185 \mathrm{mg} / \mathrm{dl}(\mathrm{p}<0,01)$, y en LDL-colesterol $\leq 100 \mathrm{mg} /$ $\mathrm{dl}$ (con $\mathrm{p}<0,001)$, y en MAU $\geq 20 \mathrm{mg} / \mathrm{l}(\mathrm{p}<0,001)$. El porcentaje de pacientes con obesidad (IMC $\geq$ $30 \mathrm{Kg} / \mathrm{m}^{2}$ ) ha disminuido del $60,9 \%$ en 2010 al $52,5 \%$ en $2014(p<0,05)$ (figura 1). Los pacientes con $\mathrm{HbA} 1 \mathrm{c} \leq 7 \%$ y con un nivel de LDL-colesterol $\leq 100 \mathrm{mg} / \mathrm{dl}$ han pasado de un $13,1 \%$ en 2010 a un $21,7 \%$ en 2014 ( $p<0,01)$, y cuando además la PA sistólica es $\leq 140 \mathrm{mmHg}$ aumenta del $8,1 \%$ en 2010 al 13,9\% en 2014 ( $p<0,05)$.

El uso del tratamiento antidiabético ha cambiado: ha disminuido el porcentaje de pacientes en tratamiento con dieta exclusivamente y el uso de glitazonas y ha aumentado el uso de metformina de $50,1 \%$ en 2010 al $57,6 \%$ en 2014 , no siendo significativo en ninguno de ellos. Por el contrario es significativo el descenso de uso de sulfonilureas: del $27,6 \%$ en 2010 a 19,0\% en 2014 ( $p<0,01$ ), así como el uso de inhibidores de la dipeptidilpeptidasa-4 (IDDP-4), que era indicado en 2010 en el $12,4 \%$ y ha pasado en estos cuatro años al $27,9 \%$ (diferencia de medias 15,5\% [IC95\%: 9,4 - 21,6]; p $<0,001$ ), lo que supone un incremento del $125 \%$ (figura 2). Los más recientes inhibidores del cotransportador de glucosa tipo 2 dependiente del sodio (SGLT-2) se están empleando aunque en un pequeño porcentaje de pacientes $(0,60 \%)$, así como los agonistas del receptor del péptido similar al glucagón de tipo 1 (GLP-1ar). El número de fármacos antidiabéticos se ha incrementado ligeramente de 1,60 (DE: 0,72) en 2010 a 1,63 (DE: 0,79) en 2014 ( $p>0,05)$.

El empleo de otros fármacos tales como hipolipemiantes se ha modificado con aumento de uso en estatinas $(44,8 \%$ en 2010 vs $53,7 \%$ en 2014 ; p < $0,05)$ y ezetimiba y descenso en fibratos. El ácido acetilsalicílico ha experimentado un incremento 


\begin{tabular}{|c|c|c|c|}
\hline Parámetro & $2010(n=297)$ & $2014(n=337)$ & $\mathbf{p}$ \\
\hline Prevalencia (IC95\%) & $8,48 \%(8,03-8,94)$ & $9,89 \%(9,41-10,39)$ & $<0,001$ \\
\hline \multicolumn{4}{|l|}{ Edad } \\
\hline Años: media (DE) & $69,48(11,1)$ & $68,75(11,07)$ & NS \\
\hline 65 años o más (IC95\%) & $69,7 \%(64,1-74,8)$ & $65,6 \%(60,2-70,6)$ & NS \\
\hline \multicolumn{4}{|c|}{ Factores de riesgo cardiovascular (IC95\%) } \\
\hline Hipertensión arterial & $73,7 \%(68,3-78,6)$ & $71,2 \%(66,0-75,9)$ & NS \\
\hline Dislipemia & $56,2 \%(50,4-61,9)$ & $54,9 \%(49,4-60,3)$ & NS \\
\hline Obesidad & $57,6 \%(51,7-63,3)$ & $54,6 \%(49,1-60,0)$ & NS \\
\hline Tabaquismo & $13,8 \%(10,1-18,3)$ & $13,0 \%(9,6-17,1)$ & NS \\
\hline \multicolumn{4}{|l|}{ Afectación orgánica (IC95\%) } \\
\hline Cardiopatía isquémica & $12,5 \%(8,9-16,7)$ & $11,3 \%(8,1-15,2)$ & NS \\
\hline Arteriopatía periférica & $8,0 \%(5,2-11,8)$ & $5,6 \%(3,4-8,6)$ & NS \\
\hline Enfermedad cerebrovascular & $9,4 \%(6,4-13,3)$ & $6,8 \%(4,4-10,0)$ & $<0,001$ \\
\hline Retinopatía diabética & $4,4 \%(2,3-7,3)$ & $2,4 \%(1,0-4,6)$ & NS \\
\hline Nefropatía diabética & $14,1 \%(10,4-18,6)$ & $8,6 \%(5,8-12,1)$ & $<0,05$ \\
\hline Neuropatía diabética & $8.8 \%(5,8-12,5)$ & $2,4 \%(1,0-4,6)$ & $<0,001$ \\
\hline \multicolumn{4}{|l|}{ Datos clínico-analíticos } \\
\hline IMC (Kg/m2): media (DE) & $31,8(5,1)$ & $31,7(14,4)$ & NS \\
\hline Glucemia (mg/dl): media (DE) & $139,6(38,9)$ & $141,0(45,1)$ & NS \\
\hline HbA1c: media (DE) & $7,22(1,21)$ & $7,09(1,17)$ & NS \\
\hline $\mathrm{HbA} 1 \mathrm{c} \geq 8 \%$ (IC95\%) & $25,9 \%(21,0-31,3)$ & $18,4 \%(14,4-22)$, & $<0,05$ \\
\hline $\mathrm{HbA1c} \geq 10 \%$ (IC95\%) & $8,1 \%(5,2-11,7)$ & $3,3 \%(1,6-5,7)$ & $<0,01$ \\
\hline Colesterol total (mg/dl): media (DE) & $195,8(38,6)$ & $183,5(37,0)$ & $<0,001$ \\
\hline LDL-colesterol (mg/dl): media (DE) & $119,4(32,3)$ & $104,2(31,4)$ & $<0,001$ \\
\hline HDL-colesterol (mg/dl): media (DE) & $47,7(10,5)$ & $50,7(14,4)$ & $<0,01$ \\
\hline Triglicéridos (mg/dl): media (DE) & $147,3(77,6)$ & $143,4(79,9)$ & NS \\
\hline PA sistólica (mmHg): media (DE) & $134,9(14,8)$ & $136,5(15,6)$ & NS \\
\hline PA diastólica (mmHg): media (DE) & $76,2(8,4)$ & $75,9(10,6)$ & NS \\
\hline Creatinina (mg/dl): media (DE) & $0,98(0,47)$ & $0,94(0,37)$ & NS \\
\hline MAU (mg/l): media (DE) & $27,8(200,1)$ & $17,8(59,9)$ & $<0,01$ \\
\hline ERC grado 1-2 + MAU (IC95\%) & $5,7 \%(3,3-9,0)$ & $6,2 \%(3,9-9,3)$ & NS \\
\hline ERC grado 3 (IC95\%) & $26,6 \%(21,6-32,0)$ & $20,8 \%(16,6-25,5)$ & NS \\
\hline ERC grado 4 (IC95\%) & $2,4 \%(0,9-4,8)$ & $1,2 \%(0,3-3,0)$ & NS \\
\hline ERC grado 5 (IC95\%) & $0,7 \%(0,01-2,4)$ & $0,3 \%(0,01-1,6)$ & NS \\
\hline Total ERC (IC95\%) & $35,4 \%(29,9-41,0)$ & $28,5 \%(23,7-33,6)$ & 0,06 \\
\hline
\end{tabular}

DE: Desviación estándar. IC95\%: Intervalo de confianza al 95\%. NS: Diferencia estadísticamente no significativa HbA1c: Hemoglobina glicosilada. MAU: microalbuminuria (de una micción). ERC: Enfermedad renal crónica

Tabla 1. Comparación de las características principales de las muestras. 


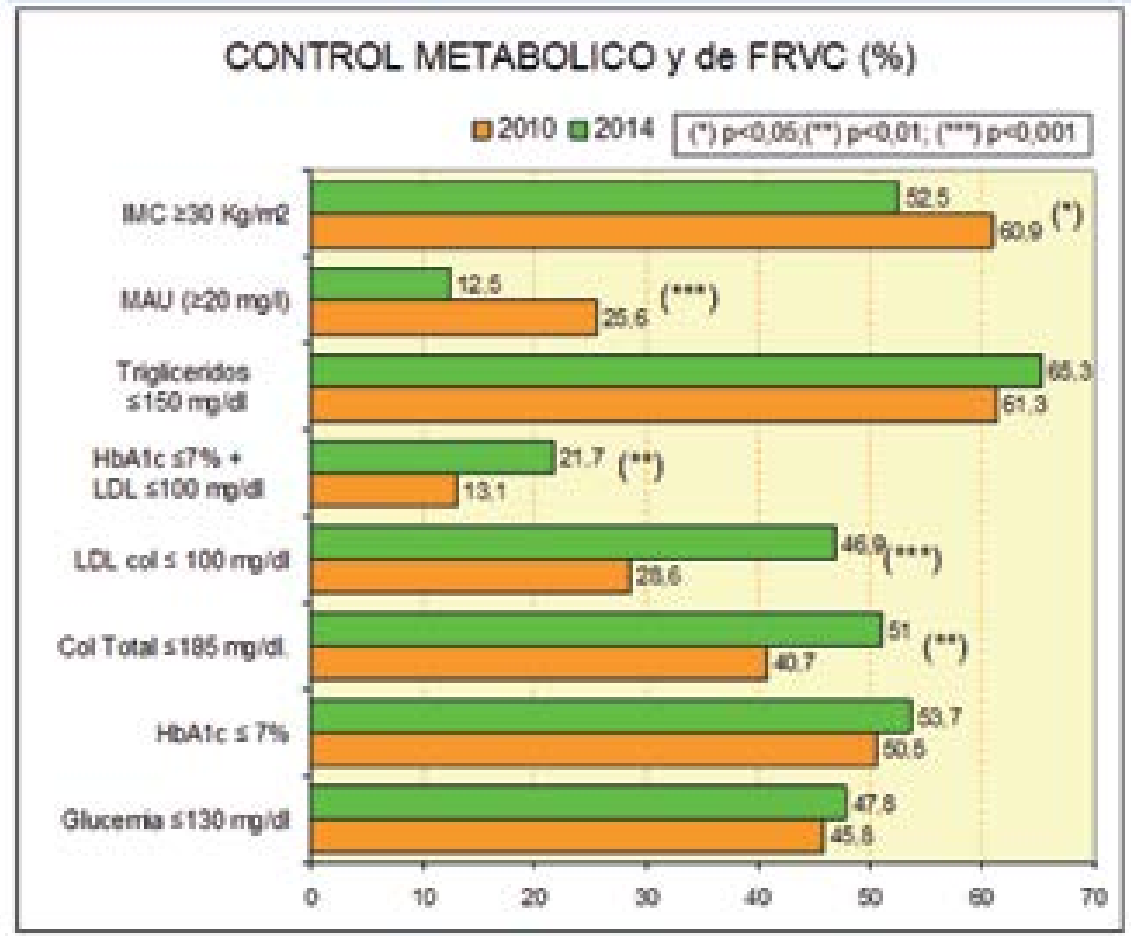

Figura 1. Control metabólico y de los factores de riesgo cardiovascular. Comparativa 2010-2014.

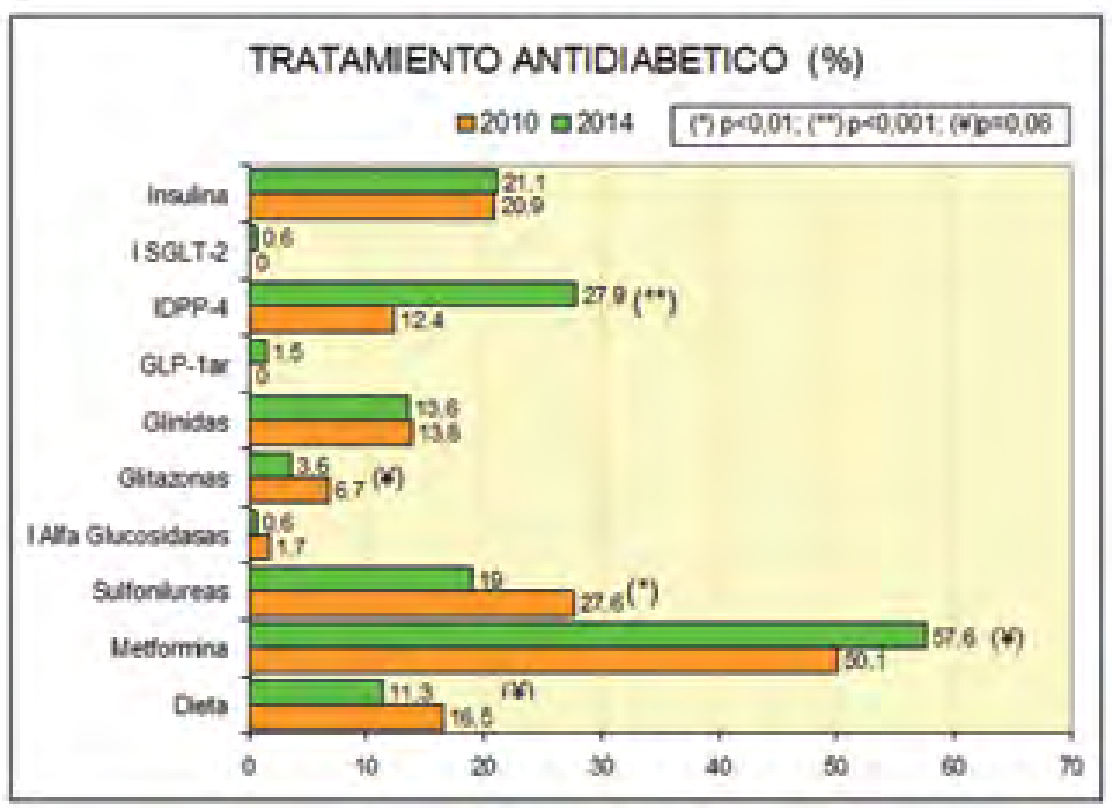

Figura 2. Variaciones en el tratamiento antidiabético, incluido dieta (2010-2014).

significativo del 40,7\% en 2010 al $51,0 \%$ en 2014 ( $p<0,01$ ). Clopidogrel, acenocumarol y otros (triflusal...) no han tenido variaciones significativas (figura 3).

\section{DISCUSIÓN}

En el periodo de cuatro años ha aumentado la prevalencia de DM2 conocida en un $1,41 \%$, pero es seguro que aún deben quedar diabéticos no conocidos. El porcentaje de diabéticos de 65 y más 


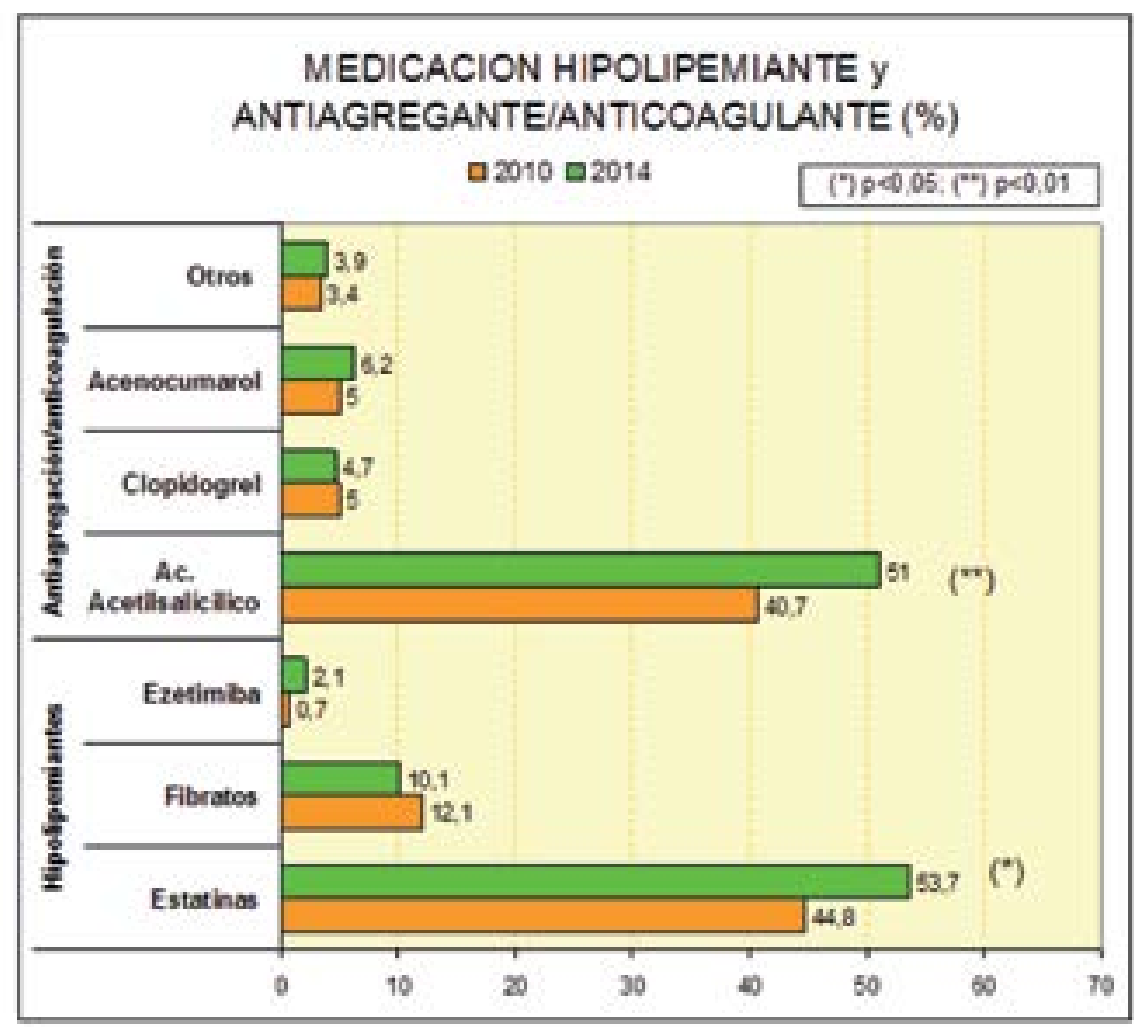

Figura 3. Uso de medicación hipolipemiante y antiagregante / anticoagulante (2010-2014).

años es menor en 2014, existiendo una menor prevalencia de los factores de riesgo cardiovascular y un menor porcentaje de pacientes con daño orgánico conocido, lo que podría deberse a un diagnóstico más temprano de la enfermedad, siendo la diabetes conocida y tratada antes de que haya podido ocasionar lesiones macro y microvasculares.

Ha mejorado el control metabólico de los pacientes con DM2 de nuestro Centro de Salud en 2014 respecto a 2010. Hay que considerar que no se trata de una cohorte de diabéticos -como otros estu$\operatorname{dios}^{21}-$, ni pacientes atendidos en un Servicio de Endocrinología como el estudio de Comi-Díaz et $\mathrm{a}^{23}$, sino de los diabéticos existentes en el Centro de Salud en cada uno de los periodos de estudio. Se detecta una mejoría en el control de la hemoglobina glicada, con valores en 2014 similares a otros estudios $^{8,24}$, y en el porcentaje de pacientes con valores elevados ( $\geq 8 \%$ y sobre todo $\geq 10 \%$ ) siendo inferiores a estudios como el de Vinagre et al ${ }^{8}$.

Las cifras medias de presión arterial sistólica se han incrementado, mientras que la presión arterial diastólica ha disminuido ligeramente (ambas sin significación estadística). Donde sí se aprecia una disminución significativa es en el patrón lipídico: mayor en LDL-colesterol, con un porcentaje de pacientes con LDL-colesterol $\leq 100 \mathrm{mg} / \mathrm{dl}$ de $46,9 \%$, superior a otros estudios ${ }^{8,11,24}$. Se ha incrementado en un $65 \%$ (del $13,1 \%$ al $21,7 \%$ ) el porcentaje de pacientes con $\mathrm{HbA} 1 \mathrm{c} \leq 7 \%$ y $\mathrm{LDL} \leq 100 \mathrm{mg} / \mathrm{dl}$, y que son superiores a otros ${ }^{22,24}$, al igual que sucede si consideramos además la PA en cifras de control (pacientes con HbA1c $\leq 7 \%$ y con LDL $\leq 100$ mg/ dl y PA sistólica $\leq 140 \mathrm{~mm} \mathrm{Hg}$ ) que ha pasado del $8,0 \%$ en 2010 al 13,9\% en 2014 ( $p<0,05)$. La prevalencia de MAU ( $\geq 20 \mathrm{mg} / \mathrm{l})$ ha disminuido de un $25,6 \%$ (en 2010) a un 12,5\% (en 2014), y el porcentaje de pacientes con obesidad ha disminuido del $60,9 \%$ al $52,5 \%$.

En el aspecto terapéutico de la DM2 se han producido modificaciones, pero sólo son significativas en la disminución de uso de sulfonilureas y en el incremento del empleo de los IDDP-4. Estas variaciones no indican a priori que se hayan intercambiado los grupos terapéuticos, ya que el descenso de sulfonilureas es inferior al aumento de IDDP-4, sino que se emplean éstas en un mayor número de pacien- 
tes, y ello aunque no son fármacos más potentes que los antidiabéticos orales previamente existentes sí presentan la ventaja de un bajo índice de hipoglucemias y a falta de estudios más exhaustivos hay una tendencia a mejorar el control glucémico con estos fármacos ${ }^{25}$. El resto de fármacos antidiabéticos no han presentado modificaciones significativas: descenso en glitazonas e incremento en metformina y dieta.

Existe también un incremento en el uso de estatinas y ácido acetilsalicílico, que en comparación con otros estudios son superiores ${ }^{22}$ o similares ${ }^{24}$.

Por tanto, se puede concluir que el control metabólico de los pacientes diabéticos tipo 2 de nuestro Centro de Salud ha mejorado, siendo mayores en el control lipídico que en el glucémico, manteniéndose el control tensional. Se ha modificado el uso de antidiabéticos orales, con disminución del porcentaje de pacientes con dieta exclusivamente, o con sulfonilureas, y aumentando el uso de IDPP4. Se ha incrementado el uso de estatinas y de antiagregación (sobre todo de ácido acetilsalicílico). Es aconsejable recordar que los objetivos de control deben ser individualizados ${ }^{26}$, y que actuar sobre el control tensional y de los valores lipídicos conseguirá resultados más exitosos a corto plazo que el control de la hiperglucemia desde el punto de vista cardiovascular ${ }^{27}$. Se ha de tener en cuenta la existencia de comorbilidades ${ }^{20}$, y la seguridad de los hipoglucemiantes no insulínicos, ya que algunos podrían ser hasta perjudiciales desde el punto de vista de la seguridad cardiovascular ${ }^{28}$.

Finalmente hemos de considerar ciertas limitaciones: a) es una comparación de dos estudios transversales separados en su realización por cuatro años, y no un estudio sobre una cohorte de pacientes con diabetes mellitus tipo 2 como el estudio de Llamazares Iglesias et $\mathrm{al}^{21}$; b) los datos analizados son de las historias clínicas cuyos datos se han considerado "válidos", y hay que tener en cuenta que el $23,4 \%$ de las historias seleccionadas han sido excluidas por falta de datos, y ello a pesar de haber realizado un proyecto de mejora asistencial a diabéticos, que si bien ha mejorado de modo global el registro de datos en un $8,7 \%$ no ha sido todo lo efectivo que sería deseable.

\section{CONFLICTO DE INTERESES}

El autor manifiesta la inexistencia de conflicto de intereses en la realización del estudio, que se ha llevado a cabo sin financiación externa y con independencia de la industria farmacéutica.

\section{BIBLIOGRAFÍA}

1. Ballester Herrera MJ, Muñoz Menor A, Giralt Contreras $P$, Racionero Camargo J, Palomo Atance E, Giralt Muiña P. Análisis del control del paciente diabético en el área de atención primaria Mancha-Centro de Castilla-La Mancha. Barómetro de la diabetes. Av Diabetol. 2012; 28 (4): 89-94.

2. Giralt Muiña P, Gutiérrez Ávila G, Ballester Herrera MJ, Botella F, Angulo JJ, el Grupo de Epidemiología de Diabetes de Castilla-La Mancha (GEDCAM). Prevalencia de diabetes y diabetes oculta en adultos de Castilla-La Mancha. Med Clin (Barc). 2011; 137 (11): 484-90.

3. Soriguer F, Goday A, Bosch-Comas A, Bordiú E, Calle-Pascual A, Carmena R et al. Prevalence of diabetes mellitus and impaired glucose regulation in Spain: the Di@bet.es Study. Diabetologia. 2012; 55 (1): 88-93.

4. Valdés S, Rojo-Martínez G, Soriguer F. Evolución de la prevalencia de la diabetes tipo 2 en población adulta española. Med Clin (Barc). 2007; 129 (9): 352-5.

5. Sicras-Mainar A, Navarro-Artieda R, Ibáñez-Molla J. Características clínicas y económicas asociadas a la diabetes tipo 2. Rev Clin Esp. 2014; 214 (3): 121-30.

6. Winocour PH. Effective diabetes care: a need for realistic targets. BMJ. 2002; 324 (7353): 1577-80.

7. Vinagre Torres I, Conget Donlo. Situación actual del control de la diabetes mellitus tipo 2 en España. Identificación de las principales barreras en la práctica clínica. Med Clin (Barc). 2013; 141 (Supl 2): 3-6.

8. Vinagre I, Mata-Cases M, Hermosilla E, Morros R, Fina F, Rosell $\mathrm{M}$ et al. Control of glycemia and cardiovascular risk factors in patients with type 2 diabetes in Primary Care in Catalonia (Spain). Diabetes Care 2012; 35 (4); 774-9.

9. Domínguez Sánchez-Migallón $\mathrm{P}$, en representación del Grupo Azuer. Control Metabólico en diabéticos tipo 2. Grado de control y nivel de conocimientos (Estudio AZUER). Rev Clin Med Fam. 2011; 4 (1): 32-41.

10. Díaz Grávalos GJ, Palmeiro Fernández G, Casado Górriz I, Arandia García M, Portuburu Izaguirre MM, Vázquez Fernández LA. Cumplimiento de los objetivos de control metabólico en diabetes mellitus en el medio rural en Ourense. Rev Esp Salud Pública. 2006; 80 (1): 67-75.

11. Díez Porres L, Riart Solans M, Foix Oña M, Morilla L, Mitjana Isarn R, Salvador Milian E et al. Control integral de los factores de riesgo cardiovascular en diabetes tipo 2 en dos comarcas rurales. Rev Clin Esp. 2010; 210 (7): 332-7.

12. Galiana Gómez del Pulgar J, en representación del Grupo ELIPSE. Efectividad en el control de factores de riesgo cardiovascular en diabéticos tipo 2 de la provincia de Ciudad Real. Rev Clin Esp. 2005; 205 (5): 218-22.

13. Jurado J, Ybarra J, Solanas P, Caula J, Gich I, Pou JM et al. Prevalence of cardiovascular disease and risk factors in a type 2 diabetic population of the North Catalonia diabetes study. J Am Acad Nurse Pract. 2009; 21 (3): 140-8.

14. Orozco-Beltrán D, Gil-Guillen VF, Quirce F, Navarro-Pérez 
J, Pineda M, Gómez-de-la Cámara A et al; Collaborative Diabetes Study Investigators. Control of diabetes and cardiovascular risk factors in patients with type 2 diabetes in primary care. The gap between guidelines and reality in Spain. Int J Clin Pract. 2007; 61 (6): 909-15.

15. Guerra Bobo A, Cañizo Fernández-Roldán C, Rovira Los$\cos$ A. Prevalencia, grado de control y tratamiento de los factores de riesgo cardiovascular en diabéticos tipo 2 asistidos en un centro de atención primaria. Av Diabetol. 2007; 23 (2): 131-6.

16. American Diabetes Association. Standards of medical care in diabetes - 2014. Diabetes Care. 2014; 37 (Suppl 1): S1480.

17. Domínguez Sánchez-Migallón P, Zamora Martin D. Aplicación de los Criterios "START" a ancianos diabéticos. XII Congreso de Atención Primaria de Castilla-La Mancha. Cuenca 12-14 Mayo de 2011.

18. Galván-Banqueri M, González-Méndez Al, Alfaro-Lara E, Nieto-Martín MD, Pérez-Guerrero C, Santos-Ramos B. Evaluación de la adecuación del tratamiento farmacológico en pacientes pluripatológicos. Aten Primaria. 2013; 45 (5): 235-43.

19. Gaede P, Lund-Andersen $\mathrm{H}$, Parving $\mathrm{HH}$, Pedersen $\mathrm{O}$. Effect of a multifactorial intervention on mortality in type 2 diabetes. N Engl J Med. 2008; 358 (6): 1577-89.

20. Tschöpe D, Hanefeld M, Meier J, Gitt A, Halle M, Bramlage $P$ et al. The role of co-morbidity in the selection of antidiabetic pharmacotherapy in type-2 diabetes. Cardiovasc Diabetol. 2013; 12: 62.

21. Llamazares Iglesias $O$, Sastre Marcos J, Peña Cortés V, Luque Pazos A, Cánovas Gaillemin B, Vicente Delgado A et al. Control metabólico y de factores de riesgo cardiovascular en una cohorte de pacientes con diabetes mellitus. Resul- tados a los 4 años. Endocrinol Nutr. 2012; 59 (2): 117-24.

22. Roca-Rodríguez M, Carral-San Laureano F, Baena-Nieto G, Aguilar-Diosdado M. Evaluación del grado de consecución de objetivos de control metabólico en pacientes con diabetes mellitus tipo 2. Endocrinol Nutr. 2010; 57 (9): 4349.

23. Comi-Díaz C, Miralles-García JM, Cabrerizo L, Pérez M, Masramon X, De Pablos-Velasco P y por el Grupo de Investigadores del Estudio Melodía de la Sociedad Española de Endocrinología y Nutrición. Grado de control metabólico en una población diabética atendida en servicios de endocrinología. Endocrinol Nutr. 2010; 57 (10): 472-8.

24. Arrieta $F$, Salinero M, Piñera M, Botella-Carretero JI, Iglesias $\mathrm{P}$, Abanades $\mathrm{JC}$ et al, en representación del grupo ESD2. Estudio descriptivo de la evolución clínico-asistencial de la población con diabetes tipo 2 en la Comunidad de Madrid. Estudio de seguimiento diabético tipo 2 (ESD-2). Av Diabetol. 2011; 27 (2): 53-60.

25. Villa Arteaga $P$, Leal Hernández $M$, Abellán Alemán J. ¿Mejora el control glucémico de los pacientes diabéticos tras la introducción de los inhibidores de la dipeptidil peptidasa 4 en su tratamiento? Aten Primaria. 2013; 45 (5): 283-4.

26. Formiga F, Pérez-Maraver M. La diabetes mellitus tipo 2 en el paciente anciano. Es básico saber cómo individualizar [editorial]. Med Clin (Barc). 2014; 142 (3): 114-5.

27. Gómez Huelgas R, Díez-Espino J, Formiga F, Lafita Tejedor J, Rodríguez Mañas L, González-Sarmiento E et al, en nombre del Grupo de Trabajo para el Documento de Consenso sobre el tratamiento de la diabetes tipo 2 en el paciente anciano. Med Clin (Barc). 2013; 140 (3): 134.e1-e12.

28. Prieto MA, Comas Samper JM, Escobar Cervantes C, Gasull Molinera V. Seguridad cardiovascular de los antidiabéticos no insulínicos. Posicionamiento científico SEMERGEN. Semergen. 2014; 40 (5): 261-73. 